\title{
Analysis of spatio-temporal variations in stroke incidence and case-fatality in Hong Kong
}

\author{
Pui Hing Chau ${ }^{1}$, Jean Woo ${ }^{1,2}$, William B. Goggins ${ }^{3}$, Moses Wong ${ }^{2}$, Kam Che Chan ${ }^{1}$, \\ Suzanne C. $\mathrm{Ho}^{4}$ \\ ${ }^{1}$ Faculty of Social Sciences, The University of Hong Kong, Hong Kong SAR, People's Republic of China; \\ ${ }^{2}$ Department of Medicine and Therapeutics, Faculty of Medicine, The Chinese University of Hong Kong, Hong \\ Kong SAR, People's Republic of China; ${ }^{3}$ Division of Biostatistics, School of Public Health and Primary Care, \\ The Chinese University of Hong Kong, Hong Kong SAR, People's Republic of China; ${ }^{4}$ School of Public Health \\ and Primary Care, The Chinese University of Hong Kong, Hong Kong SAR, People's Republic of China
}

\begin{abstract}
Geographical variations in stroke incidence and case-fatality have been documented worldwide. This study examines whether there are spatio-temporal variations in stroke incidence and case-fatality in Hong Kong and attempts to determine to what extent socioeconomic status (SES) and healthcare provision account for these variations. Residence-based hospital discharge data from the Hospital Authority (HA) in Hong Kong were geo-referenced and used to examine incidence rates and case-fatality rates by stroke subtype among the population aged 35 years and above in 1999-2007. Multilevel models were used to examine the spatio-temporal variations. Ischemic stroke incidence was found to decrease among those aged above 55 years, while hemorrhagic stroke incidence increased. Ischemic stroke case-fatality was found to decrease but hemorrhagic stroke case-fatality remained stable. For both subtypes, there were significant variations in stroke incidence and case-fatality across the districts of residence, but insignificant variations across HA service clusters. Only variations in ischemic stroke incidence and hemorrhagic stroke case-fatality at the district level could be partly explained by district-level SES. Identification of districts with higher risk for stroke incidence and case-fatality would help to formulate enhanced preventive measures. Future studies are needed to identify factors that contribute to the geographical variations.
\end{abstract}

Keywords: geographical variation, stroke, incidence, case-fatality, Hong Kong.

\section{Introduction}

Worldwide evidence shows that there are geographical variations in stroke incidence and case-fatality (Feigin et al., 2003; Ovary et al., 2004; El-Saed et al., 2008; Havulinna et al., 2008; Allen et al., 2010). Researchers have attempted to explain these geographical variations in terms of variations in socioeconomic status (SES) (Chang et al., 2002; Brown et al., 2005; Lisabeth et al., 2006; Thrift et al., 2006) and stroke risk factors (Engstrom et al., 2001; Lawlor et al., 2003; Morris et al., 2003; Voeks et al., 2008; Maheswaran et al., 2010). A historical study has explained the geographical variations in stroke incidence in China in terms of stroke risk factors (He et al., 1995) but recent epidemiological studies conducted in the Chinese population are lacking. Although

\footnotetext{
Corresponding author:

Pui Hing Chau

Room 104, Meng Wah Complex

University of Hong Kong

Pokfulam Road, Hong Kong, People's Republic of China

Tel. +852 2219 4274; Fax +852 29864596

E-mail: phchau@graduate.hku.hk
}

variations of stroke incidence in three large Chinese cities have been studied in the last few years, the main objective was to demonstrate the effect of communitybased intervention measures (Jiang et al., 2006; Wang et al., 2007).

Hong Kong, a major Asian city located on the south-eastern coast of China with a population of 7 million, has about $2 \%$ prevalence of stroke among the population aged 35 years and above as reported by the Department of Health, Hong Kong Special Administrative Region (SAR) Government and Department of Community Medicine, The University of Hong Kong (2005) and stroke is the fourth leading cause of mortality (Hospital Authority, 2010). Different trends in hemorrhagic stroke incidence and ischemic stroke incidence have been documented during the period 1999-2007 (Chau et al., 2011). However, few studies have focused on spatial and temporal patterns of stroke incidence and case-fatality in Hong Kong. Using historical mortality registration data from 1970s to the mid 1990s, Lloyd et al. (1996) documented spatial and temporal patterns of stroke mortality, but without attempting to explain the variations. 
It is uncertain whether the geographical variations are related to the provision of health services or to other characteristics of the area of residence. In Hong Kong, the territory is divided into four distinctive areas: the New Territories, Kowloon, Hong Kong Island and the outlying islands. Within these main areas, there are 18 administrative District Councils (DC). The demographic and socioeconomic profiles vary across districts (Census and Statistics Department, Hong Kong SAR Government, 2007). Since 2002, the hospitals and institutions run by the Hong Kong Hospital Authority (HA) have been grouped into seven geographical clusters (HA clusters) for local healthcare planning, financing and service delivery.

This study is the first examining recent spatio-temporal variations in incidence and case-fatality of stroke in Hong Kong, with an attempt to explain the spatial variations in terms of socioeconomic characteristics and healthcare provisions.

\section{Materials and methods}

\section{Data}

Data were obtained from the Clinical Management System database of HA to examine the incidence and case-fatality rates by stroke subtype among the Hong Kong population aged 35 years and above for the period of 1 January 1999 to 31 December 2007. Information on age, sex, district of residence, managing hospital, principal diagnosis, and dates of admission, discharge and death were extracted from the database. Details of the database have been presented elsewhere (Chau et al., 2011). Ethics approval was obtained from the ethical committees of The Chinese University of Hong Kong.

A stroke episode was defined as a hospital admission with a principal diagnosis of stroke (International Classification of Diseases, $9^{\text {th }}$ version (ICD-9), codes 430 to 438). In case of previous hospitalization, the episode would be counted as separate only if it occurred $>30$ days after the previous discharge from the hospital. For the purpose of this study, incidence was defined as stroke hospitalization episodes and case-fatality was defined as death of the patient within 30 days of hospitalization. For subtype analysis, hemorrhagic stroke was defined by ICD- 9 codes 431 and 432 , and ischemic stroke by ICD-9 codes 433, 434, 436 and 437 in the principal diagnoses of the first admission within an episode. The patients were classified into six age groups: 35-44, 45-54, 55-64, 65 $74,75-84$ and $\geq 85$ years. Three time periods, defined by the year of hospital admission (1999-2001, 20022004 and 2005-2007), were used in the analyses. Stroke episodes in the database were geo-coded into DC districts and HA clusters according to the patients' district of residence and the hospital they were admitted to, respectively.

\section{Statistical analysis}

For hemorrhagic stroke and ischemic stroke, agestandardized stroke incidence rates and case-fatality rates were calculated for the $18 \mathrm{DC}$ districts over the three periods, with the Hong Kong population and the total number of stroke cases as the standard populations, respectively. Maps were produced using ArcMap, 9.2 (ESRI, Redlands, CA, USA) for graphical illustration.

As there are two spatial classifications for the same stroke episode, namely district-level and cluster-level, cross classification models were adopted (Goldstein, 2003). Poisson link and logistic link were used to model the spatio-temporal variations in stroke incidence and case-fatality, respectively. The models were run for two stroke subtypes (hemorrhagic and ischemic) separately. The model for the stroke incidence was:

$$
\log \left(y_{i j k(l m)}\right)=\log \left(n_{i j k(l)}\right)+\Lambda_{i j k(l m)}+u_{1 l}+u_{2 m}+\mathrm{e}_{i j k(l m)}
$$

where $y_{i j k(l m)}$ denotes the number of stroke cases of the $i$-th age-group, $j$-th gender, $k$-th period, $l$-th DC district and $m$-th HA cluster; and the offset variable $n_{i j k(l)}$ is the population of the $i$-th age-group, $j$-th gender, $k$ th period and $l$-th DC district obtained from the Hong Kong Census and Statistics Department. Level 1 random structure was defined by the independently distributed variables $e_{i j k(l m)}$ and level 2 variations were resulted from the sum of random variables $u_{1 l}$ (random effect for DC district) and $u_{2 m}$ (random effect for HA cluster). $\Lambda_{i i k(m)}$ denotes the vector of fixed effects, which were all categorical variables, to be examined.

Similarly, the model for the stroke case-fatality was:

$$
\log \left(\frac{p_{i j k l(m)}}{1-p_{i j k l(l m)}}\right)=\Lambda_{i j k l(m)}+u_{1 l}+u_{2 m}+\mathrm{e}_{i i k l(m)}
$$

where $p_{i j k(l m)}$ denotes the probability of death within 30 days of hospitalization of the $i$-th age-group, $j$-th gender, $k$-th period, $l$-th DC district and $m$-th HA cluster. Other notations were the same as the Poisson model.

Individual-level fixed effects to be examined included age group, sex, period, as well as the interactions 
between age and sex, age and period, and sex and period. If significant district-level variations would be observed, district-level fixed effects -SES characteristics and primary care provision of the district- would be examined to explain the variations. The two profiles were differentiated depending on whether the median household income of the district was in the upper quartile (i.e. >HK\$ 20,000) and whether the number of public primary care clinics per 100,000 population in that district was above the mean of that in Hong Kong as a whole (i.e. 1.08 per 100,000 population), using the year 2006 as reference. Since the SES indicator of educational level was highly correlated with that of income, only the household income was included as SES indicator. If there were significant cluster-level variations, explanations would be attempted by examining the public hospital resources of the HA clusters, by using the following indicators: (i) whether or not the medical doctors-to-population ratio was above the local mean (i.e. 0.70 per 1,000 population); (ii) whether or not beds-to-population ratio was above the local mean (i.e. 3.96 per 1,000 population); (iii) whether or not $>40 \%$ of stroke patients were treated in acute stroke units; and (iv) whether or not $>80 \%$ of stroke patients had received a CT scan, again using the year 2006 as reference.

Data analysis was performed with SAS, Release 9.2 (SAS Institute, Inc, Cary, NC, USA) and the multilevel models with the PROC GLIMMIX procedure. A significance level of 0.05 was used in all analyses.

\section{Results}

\section{Spatial and temporal patterns}

From 1999 to 2007, 26,888 hemorrhagic stroke episodes and 108,436 ischemic stroke episodes were identified from the HA database in Hong Kong, where $97.8 \%$ of the hemorrhagic stroke and $99.3 \%$ of the ischemic stroke cases were geo-referenced. Fig. 1 and Fig. 2 show the geographic distribution of age-standardized stroke incidence and stroke case-fatality

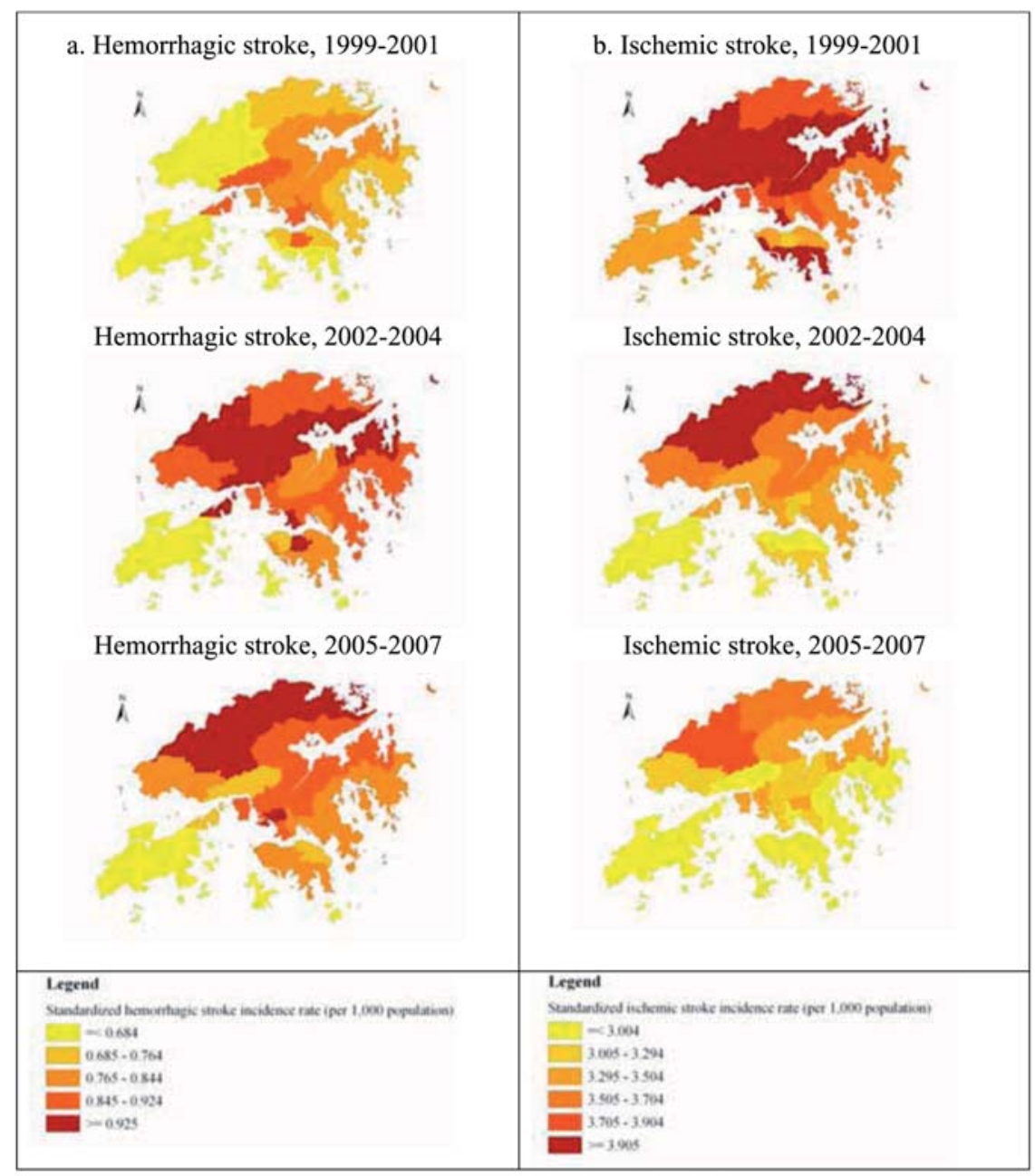

Fig. 1. Age-standardized stroke incidence rates by DC districts in Hong Kong, breakdown by stroke subtype and time period. 


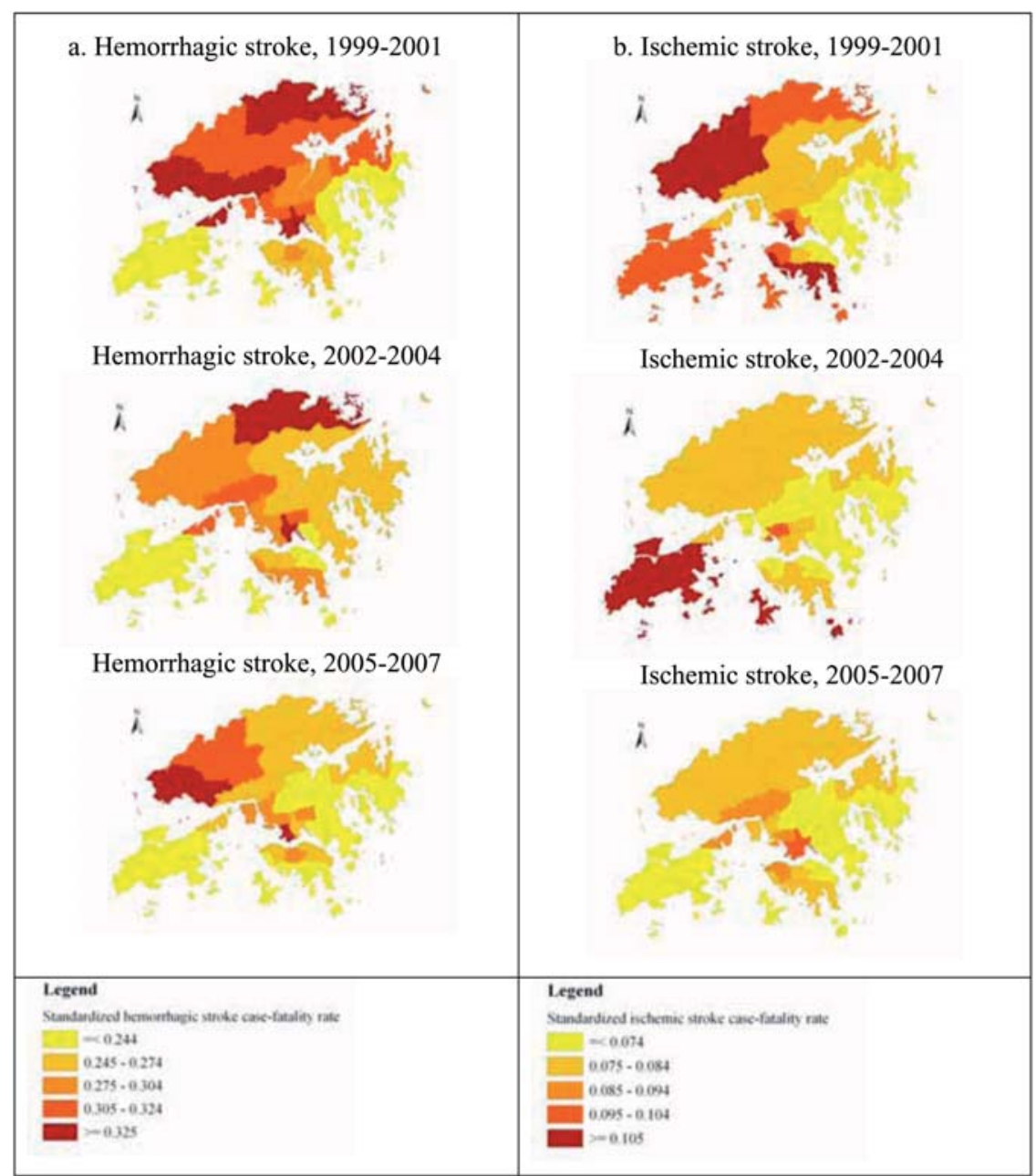

Fig. 2. Age-standardized stroke case-fatality rates by DC districts in Hong Kong, breakdown by stroke subtype and time period.

across the three time periods, respectively. Analysed by district, increasing number of districts with high hemorrhagic stroke incidence was found, while the number of districts with a high ischemic stroke incidence decreased. Meanwhile, the number of districts with high case-fatalities of both hemorrhagic and ischemic strokes decreased.

For hemorrhagic stroke, high incidence rates were seen to spread out over time from the central part of Hong Kong to other districts in the New Territories. For ischemic stroke, high incidence rates spread over the entire territory in 1999-2001, but clustered in the northern part in 2005-2007. With regard to the casefatalities (for both stroke subtypes), high rates clustered in most parts of Kowloon and the north-western parts of the New Territories across the three time periods.

\section{Multilevel models for incidence and case-fatality}

The cross classification models (with random effects only) showed that there were insignificant variations in stroke incidence and case-fatality at the HA-cluster level $(\mathrm{P}=0.446$ and $\mathrm{P}=0.557$ for hemorrhagic and ischemic stroke incidence, respectively; $\mathrm{P}=0.143$ and $\mathrm{P}=0.218$ for hemorrhagic and ischemic stroke casefatality, respectively), which was then removed from the models. On the other hand, significant district variations in stroke incidence $(\mathrm{P}=0.005$ for hemorrhagic; $\mathrm{P}=0.004$ for ischemic) and case-fatality $(\mathrm{P}=0.015$ for hemorrhagic; $\mathrm{P}=0.007$ for ischemic $)$ were observed. Hence, district-level indicators of household income and primary care availability were added to the models. Meanwhile, individual-level explanatory variables were also added.

Spatial variations in hemorrhagic stroke case-fatality and ischemic stroke incidence could be partly explained by district-level SES "proxied" by household income indicator $(\mathrm{P}=0.009$ and $\mathrm{P}=0.002$, respectively), where districts with lower household income were associated with higher rates. However, district-level SES could not explain the spatial variations in hemorrhagic stroke incidence $(P=0.136)$ and ischemic stroke 
case-fatality $(\mathrm{P}=0.330)$. Meanwhile, district-level primary care provision could not explain both the incidence $(\mathrm{P}=0.889$ for hemorrhagic; $\mathrm{P}=0.177$ for ischemic) and the case-fatality $(\mathrm{P}=0.249$ for hemorrhagic; $\mathrm{P}=0.116$ for ischemic) of both subtypes.

At the individual level, stroke incidence (both subtypes) was found to be associated with both age and sex (interaction: $\mathrm{P}<0.0001)$. This was also observed for ischemic stroke case-fatality (interaction: $\mathrm{P}=0.023$ ). Hemorrhagic and ischemic stroke incidence increased with age. Females had lower hemorrhagic stroke incidence than males, with sex effect smaller for older age groups; whereas females had lower ischemic stroke incidence than males up to the age of 85 years. Ischemic stroke case-fatality increased with age, women aged 75 years and above had higher case-fatality than men. On the other hand, higher hemorrhagic stroke case-fatality was associated with increasing age $(\mathrm{P}<0.0001)$, but there were insignificant age-sex inter- actions $(\mathrm{P}=0.066)$ and sex difference $(\mathrm{P}=0.435)$.

The incidence of hemorrhagic stroke increased in 2002-2004 and 2005-2007 compared with 19992001, with magnitude depending on the age group (interaction: $\mathrm{P}<0.0001)$. Ischemic stroke incidence trends were found to be dependent on age (interaction: $\mathrm{P}<0.0001$ ) and sex (interaction: $\mathrm{P}=0.005)$. Ischemic stroke incidence decreased among those aged above 55 years in 2002-2004 and 2005-2007 as compared with 1999-2001, but was stable among those aged 35-54 years. While ischemic stroke case-fatality decreased in 2002-2004 and 2005-2007 compared with 19992001, with magnitude being independent of age group and sex, hemorrhagic stroke case-fatality did not show significant trends.

To avoid over-fitting the models, the principle of parsimony was applied. That is, all insignificant factors and interaction terms were removed from the models. Based on the final model, Table 1 summarises

Table 1. Regression results of district variations in stroke incidence and case-fatality in Hong Kong

\begin{tabular}{|c|c|c|c|c|}
\hline \multirow{2}{*}{$\begin{array}{l}\text { District Council } \\
\text { (DC) district }\end{array}$} & \multicolumn{2}{|c|}{ Incidence } & \multicolumn{2}{|c|}{ Case-fatality } \\
\hline & Hemorrhage $^{1}$ & Ischemic $^{2}$ & Hemorrhage $^{3}$ & Ischemic $^{4}$ \\
\hline & \multicolumn{4}{|c|}{ Estimate (SE) } \\
\hline \multicolumn{5}{|l|}{ Hong Kong Island } \\
\hline Central \& Western & $-0.1067(0.0344)^{*}$ & $-0.0750(0.0417)$ & $0.0004(0.0764)$ & $0.0875(0.0656)$ \\
\hline Wan Chai & $0.0792(0.0359)^{*}$ & $-0.0893(0.0424)^{*}$ & $0.0737(0.0772)$ & $-0.0055(0.0738)$ \\
\hline Eastern & $-0.0815(0.0268)^{*}$ & $-0.0118(0.0404)$ & $-0.0497(0.0679)$ & $-0.1435(0.0534)^{*}$ \\
\hline Southern & $-0.0667(0.0327)^{*}$ & $0.1029(0.0411)^{*}$ & $0.0220(0.0742)$ & $0.1071(0.0597)$ \\
\hline \multicolumn{5}{|l|}{ Kowloon } \\
\hline Yau Tsim Mong & $0.0828(0.0309)^{*}$ & $0.0218(0.0275)$ & $0.1219(0.0599) *$ & $0.1562(0.0576)^{*}$ \\
\hline Sham Shui Po & $0.0783(0.0280)^{*}$ & $-0.0026(0.0266)$ & $0.0740(0.0546)$ & $0.1916(0.0521)^{*}$ \\
\hline Kowloon City & $0.0447(0.0289)$ & $-0.0916(0.0270)^{*}$ & $0.0768(0.0562)$ & $0.1317(0.0547)^{*}$ \\
\hline Wong Tai Sin & $0.0118(0.0273)$ & $-0.0186(0.0263)$ & $0.0671(0.0534)$ & $-0.1066(0.0533)^{*}$ \\
\hline Kwun Tong & $-0.0424(0.0263)$ & $-0.0295(0.0260)$ & $-0.2372(0.0531)^{*}$ & $-0.3462(0.0536)^{*}$ \\
\hline \multicolumn{5}{|l|}{ New Territories } \\
\hline Kwai Tsing & $0.0279(0.0273)$ & $0.0194(0.0263)$ & $0.0389(0.0539)$ & $-0.0663(0.0541)$ \\
\hline Tsuen Wan & $0.0218(0.0332)$ & $-0.1020(0.0286)^{*}$ & $0.0442(0.0646)$ & $0.0080(0.0655)$ \\
\hline Tuen Mun & $-0.0617(0.0305)^{*}$ & $0.0471(0.0271)$ & $0.1093(0.0600)$ & $0.0915(0.0572)$ \\
\hline Yuen Long & $0.0571(0.0299)$ & $0.1526(0.0269)^{*}$ & $0.0380(0.0587)$ & $0.1203(0.0551)^{*}$ \\
\hline North & $0.0391(0.0337)$ & $0.0868(0.0281)^{*}$ & $0.0148(0.0656)$ & $0.0791(0.0621)$ \\
\hline Tai Po & $0.0681(0.0332)^{*}$ & $0.0698(0.0282)^{*}$ & $-0.0587(0.0652)$ & $0.0128(0.0631)$ \\
\hline Sha Tin & $0.0269(0.0274)$ & $0.0180(0.0264)$ & $-0.1601(0.0551)^{*}$ & $-0.1010(0.0537)$ \\
\hline Sai Kung & $-0.0231(0.0335)$ & $0.0731(0.0415)$ & $-0.0465(0.0757)$ & $-0.2647(0.0703)^{* *}$ \\
\hline Islands & $-0.1557(0.0470)^{*}$ & $-0.1712(0.0349)^{*}$ & $-0.1289(0.0889)$ & $0.0482(0.0901)$ \\
\hline
\end{tabular}

\footnotetext{
${ }^{1}$ Final model for hemorrhagic stroke incidence. Random effect: district. District-level fixed effect: --. individual-level fixed effect: age, sex, period, age-sex interaction, age-period interaction. Poisson link used; ${ }^{2}$ final model for ischemic stroke incidence. Random effect: district. District-level fixed effect: household income indicator. Individual-level fixed effect: age, sex, period, age-sex interaction, age-period interaction, sex-period interaction. Poisson link used; ${ }^{3}$ final model for hemorrhagic stroke case-fatality. Random effect: district. District-level fixed effect: household income indicator. Individual-level fixed effect: age, sex, period. Logit link used; ${ }^{4}$ final model for ischemic stroke case-fatality. Random effect: district. District-level fixed effect:--. individual-level fixed effect: age, sex, period, age-sex interaction. Logit link used; $* \mathrm{P}<0.05$.
} 
the spatial variations across districts. SES could only explain $37 \%$ of the district variations in ischemic stroke incidence and $35 \%$ of district variations in hemorrhagic stroke case-fatality.

\section{Discussion}

The geographical variations in stroke incidence and case-fatality analyzed according to service provision clusters were insignificant, although an association between healthcare provisions and health disparities has been documented (Weisz et al., 2008). For stroke incidence, this is not surprising since the occurrence of stroke events is unlikely to be related to the availability of stroke care facilities or treatments. For casefatality, the results suggest that any variation in healthcare provisions may not always result in geographic variations in health outcomes. Furthermore, severity of stroke may largely determine the case-fatality irrespective of the quality or quantity of healthcare provision.

By contrast, individual lifestyle and socioeconomic position have been considered as having greater impact on health outcome (Woo et al., 2010). Our findings showed that $37 \%$ of the variations in ischemic stroke incidence at the district level could be explained by district-level SES. This agrees with previous studies showing that higher stroke incidence was associated with districts of lower SES (Chang et al., 2002; Brown et al., 2005; Lisabeth et al., 2006; Thrift et al., 2006), where health literacy may be inadequate and financial resources limited. However, district variations in hemorrhagic stroke incidence and ischemic stroke case-fatality could not be explained by SES. Even for ischemic stroke incidence and hemorrhagic stroke case-fatality, the proportion of district variations explained by SES was not large $(>40 \%)$. The findings suggest that factors other than SES, such as air pollution, living environment and psychological stress, may contribute directly to the district variations in stroke incidence and case-fatality, as well as to their risk factors (such as smoking, hypertension, overweight) independent of SES.

Identification of high-risk districts may help inform public health policy to prioritize intervention strategies to these communities. Possible risk factors other than SES that contribute to the high-risk districts may include air quality or psychological stress arising from living in very crowded environments. For example, Yau Tsim Mong (located in western Kowloon) was found to have significantly higher case-fatality for both stroke subtypes and higher hemorrhagic stroke incidence than the local average. Besides low household income, as suggested by the multilevel models, one of the possible explanations included serious roadside air pollution in this district as reported by Civic Exchange (2010). As air pollution has been found to be associated with survival after stroke (Maheswaran et al., 2010), stroke mortality (Hu et al., 2000) and stroke hospital admissions (Oudin et al., 2010), improvement in air quality might help reducing case-fatality and incidence. Moreover, stress may be associated with higher risk of fatal stroke events (Trueslen et al., 2003). Yau Tsim Mong is one of the most densely populated districts in Hong Kong, where the urban living environment is not only crowded but also busy adding extra strain. Those living in highdensity urban districts tend to suffer from more environmental nuisances and psychological stress (Chan and Lee, 2009). Increasing green space may help reducing stress levels (Woo et al., 2009) and this may have a beneficial effect on blood pressure, which is a major risk factor for stroke occurrence, particularly for haemorrhagic stroke. Since hemorrhagic stroke is more likely to be fatal, the role of increasing green space may be explained by a larger reduction in hemorrhagic than ischemic stroke, and hence result in reduction in the overall stroke fatality rate.

There was no clear consistent pattern among the 18 districts with respect to significantly lower-than-average incidence and case-fatality for both stroke subtypes. The Islands district (mainly the south-western islands and other remote islands) had lower stroke incidence for both subtypes, which may be attributable to the more relaxed lifestyle in this district. Kwun Tong (located in eastern Kowloon) had lower casefatality for both subtypes, but the reason for this is uncertain.

As this study was based on secondary data analysis, there are some limitations related to the use of the hospitalization dataset for such analysis, which have been discussed elsewhere (Chau et al., 2011). Furthermore, other factors such as medical history, individual lifestyle and work-related stress that may have contributed to the incidence and case-fatality patterns were not included as these factors were not available. Further studies are needed to investigate the mechanism and relative importance of these factors in contributing to the spatial variations.

This study demonstrates recent spatio-temporal variations in hemorrhagic and ischemic strokes incidence and case-fatality in Hong Kong, where casefatality and incidence of ischemic stroke were found to decrease, while the incidence of hemorrhagic stroke 
increased. Variations in incidence and case-fatality could not be explained by variations in service provisions at service cluster level, although variations in hemorrhagic stroke case-fatality and ischemic stroke incidence at district level could be partly explained by SES. The study identified one district with increased risk for both incidence and case-fatality, suggesting that preventive measures should be strengthened in that district. Further studies are needed to identify contributory factors to geographical variations other than SES.

\section{Acknowledgements}

This study was supported by "CADENZA: A Jockey Club Initiative for Seniors" funded by The Hong Kong Jockey Club Charities Trust, and the Health and Health Services Research Fund (HHSRF: 06070451), Food and Health Bureau, Hong Kong SAR Government. The authors would like to acknowledge the Strategy and Planning Division of the Hospital Authority for the provision of data for this study.

\section{References}

Allen NB, Holford TR, Bracken MB, Goldstein LB, Howard G, Wang Y, Lichtman J, 2010. Geographic variation in one-year recurrent ischemic stroke rates for elderly Medicare beneficiaries in the USA. Neuroepidemiology 34, 123-129.

Brown P, Guy M, Broad J, 2005. Individual socioeconomic status, community socioeconomic status and stroke in New Zealand: a case control study. Soc Sci Med 61, 1174-1188.

Census and Statistics Department, Hong Kong SAR Government, 2007. 2006 Population By-census: basic tables for district council districts. Available at: http://www.bycensus2006.gov.hk/FileManager/EN/Content_962/06bc_dcd.pdf (accessed on October 2011).

Chan EHW, Lee GKL, 2009. Design considerations for environmental sustainability in high density development: a case study of Hong Kong. Environ Dev Sustain 11, 359-374.

Chang CL, Marmot MG, Farley TMM, Poulter NR, 2002. The influence of economic development on the association between education and the risk of acute myocardial infraction and stroke. J Clin Epidemiol 55, 741-747.

Chau PH, Woo J, Goggins WB, Tse YK, Chan KC, Lo SV, Ho SC, 2011. Trends in stroke incidence in Hong Kong differ by stroke subtype. Cerebrovasc Dis 31, 138-146.

Civic Exchange, 2010. Fixing roadside pollution: matching problems with solutions, 18-district survey reveals priority areas and solutions. Available at: http://civic-exchange.org/en/live/ upload/files/20100622_MAP.pdf (accessed on October 2011).

Department of Health, Hong Kong SAR Government, Department of Community Medicine, The University of Hong
Kong, 2005. Population Health Survey 2003/2004. Available at: http://www.chp.gov.hk/files/pdf/full_report_on_population _health_survey_2003_2004_en_20051024.pdf (accessed on October 2011).

El-Saed A, Kuller LH, Newman AB, Lopez O, Costantino J, McTigue K, Cushman M, Kronmal R, 2006. Geographic variations in stroke incidence and mortality among older populations in four US communities. Stroke 37, 1975-1979.

Engstrom G, Jerntorp I, Pessah-Rasmussen H, Hedblad B, Berglund G, Janzon L, 2001. Geographic distribution of stroke incidence within an urban population: relations to socioeconomic circumstances and prevalence of cardiovascular risk factors. Stroke 32, 1098-1103.

Feigin VL, Lawes CM, Bennett DA, Anderson CS, 2003. Stroke epidemiology: a review of population-based studies of incidence, prevalence, and case-fatality in the late 20th century. Lancet Neurol 2, 43-53.

Goldstein H, 2003. Multilevel statistical models (3rd ed.). Edward Arnold, London, UK, pp. 187-198.

Havulinna AS, Paakkonen R, Karvonen M, Salomaa V, 2008. Geographic patterns of incidence of ischemic stroke and acute myocardial infarction in Finland during 1991-2003. Ann Epidemiol 18, 206-213.

He J, Klag MJ, Wu Z, Whelton PK, 1995. Stroke in the People's Republic of China. Stroke 26, 2222-2227.

Hospital Authority, 2010.. HA Statistical Report 2008-2009. Available at: http://www.ha.org.hk/upload/publication_15 1281.pdf (accessed on October 2011).

Hu Z, Liebens J, Rao KR, 2008. Linking stroke mortality with air pollution, income, and greenness in northwest Florida: an ecological geographical study. Int J Health Geogr 7, 20.

Jiang B, Wang WZ, Chen H, Hong Z, Yang QD, Wu SP, Du XL, Bao QJ, 2006. Incidence and trends of stroke and its subtypes in China: results from three large cities. Stroke 37, 63-68.

Lawlor DA, Bedford C, Taylor M, Ebrahim S, 2003. Geographical variation in cardiovascular disease, risk factors, and their control in older women: British Women's Heart and Health Study. J Epidemiol Commun Health 57, 134-140.

Lisabeth LD, Diez Roux AV, Escobar JD, Smith MA, Morgenstern LB, 2006. Neighbourhood environment and risk of ischemic stroke. Am J Epidemiol 165, 279-287.

Lloyd OL, Wong TW, Wong SL, Yu TSI, 1996. Atlas of disease mortalities in Hong Kong for the three five-year periods in 1979-93, The Chinese University Press, Hong Kong, China.

Maheswaran R, Perason T, Smeeton NC, Beevers SD, Campell MJ, Wolfe CD, 2010. Impact of outdoor air pollution on survival after stroke: population-based cohort study. Stroke 41, 869-877.

Morris RW, Whincup PH, Emberson JR, Lampe FC, Walker M, Shaper AG, 2003. North-south gradients in Britain for stroke and CHD: are they explained by the same factors? Stroke 34, 2604-2609. 
Oudin A, Stromberg U, Jakobsson K, Stroh E, Bjork J, 2010. Estimation of short-term effects of air pollution on stroke hospital admissions in southern Sweden. Neuroepidemiology 34, 131-142.

Ovary C, Suzuki K, Nagy Z, 2004. Regional differences in incidence rates, outcome predictors and survival of stroke. Neuroepidemiology 23, 240-246.

Thrift AG, Dewey HM, Sturm JW, Paul SL, Gilligan AK, Srikanth VK, Macdonell RAL, McNeil JJ, Macleod MR, Donnan GA, 2006. Greater incidence of both fatal and nonfatal strokes in disadvantaged areas: the Northeast Melbourne Stroke Incidence Study. Stroke 37, 877-882.

Truelsen T, Nielsen N, Boysen G, Gronbaek M, 2003. Selfreported stress and risk of stroke: the Copenhagen City Heart Study. Stroke 34, 856-862.

Voeks JH, McClure LA, Go RC, Prineas RJ, Cushman M, Kissela BM, Roseman JM, 2008. Regional differences in dia- betes as a possible contributor to the geographic disparity in stroke mortality: the Reasons for Geographic and Racial Differences in Stroke Study. Stroke 39, 1675-1680.

Wang WZ, Jiang B, Wu Sp, Hong Z, Yang QD, Sander JW, Du XL, Bao QJ, 2007. Change in stroke incidence from a population-based intervention trail in three urban communities in China. Neuroepidemiology 28, 155-161.

Weisz D, Gusmano MK, Rodwin VG, Neuberg LG, 2008. Population health and the health system: a comparative analysis of avoidable mortality in three nations and their world cities. Eur J Public Health 18, 166-172.

Woo J, Chan R, Leung J, Wong M, 2010. Relative contributions of geographic, socioeconomic, and lifestyle factors to quality of life, frailty, and mortality in elderly. PLoS One 5, e8775.

Woo J, Tang N, Suen E, Leung J, Wong M, 2009. Green space, psychological restoration, and telomere length. Lancet 373, 299-300. 